\title{
OVERLAPPING STOCHASTIC BLOCK MODELS WITH APPLICATION TO THE FRENCH POLITICAL BLOGOSPHERE : APPENDIX
}

\author{
By Pierre Latouche ${ }^{\dagger}$, Etienne Birmelé $^{\dagger}$ and Christophe \\ AMBroise $^{\dagger}$ \\ Laboratoire Statistique et Génome, UMR CNRS 8071, UEVE
}

${ }^{*}$ This work has been supported by the French Agence Nationale de la Recherche under grant NeMo ANR-08-BLAN-0304-01. 


\section{APPENDIX A: COMPUTATION OF THE LOWER BOUNDS}

A.1. First lower bound . The lower bound can be written:

$$
\begin{aligned}
\mathcal{L}(q ; \boldsymbol{\alpha}, \tilde{\mathbf{W}}) & =\sum_{\mathbf{Z}} q(\mathbf{Z}) \ln p(\mathbf{X}, \mathbf{Z} \mid \boldsymbol{\alpha}, \tilde{\mathbf{W}})-\sum_{\mathbf{Z}} q(\mathbf{Z}) \ln q(\mathbf{Z}) \\
& =\mathrm{E}_{\mathbf{Z}}[\ln p(\mathbf{X}, \mathbf{Z} \mid \boldsymbol{\alpha}, \tilde{\mathbf{W}})]-\mathrm{E}_{\mathbf{Z}}[\ln q(\mathbf{Z})] \\
& =\mathrm{E}_{\mathbf{Z}}[\ln p(\mathbf{X} \mid \mathbf{Z}, \tilde{\mathbf{W}})]+\mathrm{E}_{\mathbf{Z}}[\ln p(\mathbf{Z} \mid \boldsymbol{\alpha})]-\mathrm{E}_{\mathbf{Z}}[\ln q(\mathbf{Z})]
\end{aligned}
$$

where the expectations are taken according to the distribution $q(\mathbf{Z})$ and the last term of (1) is an entropy term. Since $q(\mathbf{Z})$ is assumed to be a product of Bernoulli distribution, we obtain:

$$
\begin{aligned}
& \mathcal{L}(q ; \boldsymbol{\alpha}, \tilde{\mathbf{W}})=\sum_{i \neq j}^{N}\{\left.X_{i j} \mathrm{E}_{\mathbf{Z}_{i}, \mathbf{Z}_{j}}\left[a_{\mathbf{Z}_{i}, \mathbf{Z}_{j}}\right]+\mathrm{E}_{\mathbf{Z}_{i}, \mathbf{Z}_{j}}\left[\operatorname{lng}\left(-a_{\mathbf{Z}_{i}, \mathbf{Z}_{j}}\right)\right]\right\} \\
&+\sum_{i=1}^{N} \sum_{q=1}^{Q}\left\{\mathrm{E}_{Z_{i q}}\left[Z_{i q}\right] \ln \alpha_{q}+\left(1-\mathrm{E}_{Z_{i q}}\left[Z_{i q}\right]\right) \ln \left(1-\alpha_{q}\right)\right\} \\
&-\sum_{i=1}^{N} \sum_{q=1}^{Q}\left\{\mathrm{E}_{Z_{i q}}\left[Z_{i q}\right] \ln \tau_{i q}+\left(1-\mathrm{E}_{Z_{i q}}\left[Z_{i q}\right]\right) \ln \left(1-\tau_{i q}\right)\right\} \\
&=\sum_{i \neq j}^{N}\left\{X_{i j} \tilde{\boldsymbol{\tau}}_{i}^{\top} \tilde{\mathbf{W}} \tilde{\boldsymbol{\tau}}_{j}+\mathrm{E}_{\mathbf{Z}_{i}, \mathbf{Z}_{j}}\left[\operatorname { l n } \mathrm { g } \left(-a_{\left.\left.\left.\mathbf{Z}_{i}, \mathbf{Z}_{j}\right)\right]\right\}}\right.\right.\right. \\
&+\sum_{i=1}^{N} \sum_{q=1}^{Q}\left\{\tau_{i q} \ln \alpha_{q}+\left(1-\tau_{i q}\right) \ln \left(1-\alpha_{q}\right)\right\} \\
&-\sum_{i=1}^{N} \sum_{q=1}^{Q}\left\{\tau_{i q} \ln \tau_{i q}+\left(1-\tau_{i q}\right) \ln \left(1-\tau_{i q}\right)\right\} .
\end{aligned}
$$

A.2. Second lower bound. The first lower bound is a function of the

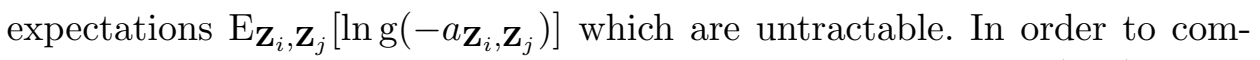
pute a second tractable lower bound, we consider the bound $\ln g(x, \xi)$ on the log-logistic function:

$$
\ln g(x) \geq \ln g(x, \xi)=\ln g(\xi)+\frac{(x-\xi)}{2}-\lambda(\xi)\left(x^{2}-\xi^{2}\right), \forall x, \xi \in \mathbb{R},
$$

where $\lambda(\xi)=\frac{1}{4 \xi} \tanh \left(\frac{\xi}{2}\right)=\frac{1}{2 \xi}\left\{\mathrm{g}(\xi)-\frac{1}{2}\right\}$ and $\xi$ is a variational parameter. This bound was first introduced by Jaakkola and Jordan (2000), in 
the framework of Bayesian logistic regression, to obtain a tractable approximation of the marginal likelihood. It is based on symmetrization of the log-logistic function and a Taylor expansion in the variable $x^{2}$. It leads to:

$$
\operatorname{lng}\left(-a_{\mathbf{Z}_{i}, \mathbf{Z}_{j}}\right)=\operatorname{lng}\left(-\tilde{\mathbf{Z}}_{i}^{\top} \tilde{\mathbf{W}} \tilde{\mathbf{Z}}_{j}\right) \geq \ln \mathrm{g}\left(-\tilde{\mathbf{Z}}_{i}^{\top} \tilde{\mathbf{W}} \tilde{\mathbf{Z}}_{j}, \xi_{i j}\right),
$$

where

$\operatorname{lng}\left(-\tilde{\mathbf{Z}}_{i}^{\top} \tilde{\mathbf{W}} \tilde{\mathbf{Z}}_{j}, \xi_{i j}\right)=\operatorname{lng}\left(\xi_{i j}\right)-\frac{\left(\tilde{\mathbf{Z}}_{i}^{\top} \tilde{\mathbf{W}} \tilde{\mathbf{Z}}_{j}+\xi_{i j}\right)}{2}-\lambda\left(\xi_{i j}\right)\left(\left(\tilde{\mathbf{Z}}_{i}^{\top} \tilde{\mathbf{W}} \tilde{\mathbf{Z}}_{j}\right)^{2}-\xi_{i j}^{2}\right)$

Therefore, we have:

$$
\begin{aligned}
& \mathrm{E}_{\mathbf{Z}_{i}, \mathbf{Z}_{j}}\left[\operatorname{lng}\left(-a_{\mathbf{Z}_{i}, \mathbf{Z}_{j}}\right)\right]=\sum_{\mathbf{Z}_{i}, \mathbf{Z}_{j} \in\{0,1\}^{Q}} \ln \mathrm{g}\left(-a_{\mathbf{Z}_{i}, \mathbf{Z}_{j}}\right) q\left(\mathbf{Z}_{i}\right) q\left(\mathbf{Z}_{j}\right) \\
& \geq \sum_{\mathbf{Z}_{i}, \mathbf{Z}_{j} \in\{0,1\}^{Q}}\left\{\operatorname{lng}\left(\xi_{i j}\right)-\frac{\left(\tilde{\mathbf{Z}}_{i}^{\top} \tilde{\mathbf{W}} \tilde{\mathbf{Z}}_{j}+\xi_{i j}\right)}{2}-\lambda\left(\xi_{i j}\right)\left(\left(\tilde{\mathbf{Z}}_{i}^{\top} \tilde{\mathbf{W}} \tilde{\mathbf{Z}}_{j}\right)^{2}\right.\right. \\
& \left.\left.-\xi_{i j}^{2}\right)\right\} q\left(\mathbf{Z}_{i}\right) q\left(\mathbf{Z}_{j}\right) \\
& \geq \ln g\left(\xi_{i j}\right)-\frac{\left(\tilde{\boldsymbol{\tau}}_{i}{ }^{\top} \tilde{\mathbf{W}} \tilde{\boldsymbol{\tau}}_{j}+\xi_{i j}\right)}{2}-\lambda\left(\xi_{i j}\right)\left(\mathrm{E}_{\mathbf{Z}_{i}, \mathbf{Z}_{j}}\left[\left(\tilde{\mathbf{Z}}_{i}^{\top} \tilde{\mathbf{W}}_{\mathbf{Z}_{j}}\right)^{2}\right]-\xi_{i j}^{2}\right) .
\end{aligned}
$$

The expectation terms are now tractable:

$$
\begin{aligned}
& \mathrm{E}_{\mathbf{Z}_{i}, \mathbf{Z}_{j}}\left[\left(\tilde{\mathbf{Z}}_{i}^{\top} \tilde{\mathbf{W}} \tilde{\mathbf{Z}}_{j}\right)^{2}\right]=\mathrm{E}_{\mathbf{Z}_{i}, \mathbf{Z}_{j}}\left[\tilde{\mathbf{Z}}_{i}^{\top} \tilde{\mathbf{W}} \tilde{\mathbf{Z}}_{j} \tilde{\mathbf{Z}}_{i}^{\top} \tilde{\mathbf{W}} \tilde{\mathbf{Z}}_{j}\right] \\
& =\mathrm{E}_{\mathbf{Z}_{i}, \mathbf{Z}_{j}}\left[\tilde{\mathbf{Z}}_{j}^{\top} \tilde{\mathbf{W}}^{\top} \tilde{\mathbf{Z}}_{i} \tilde{\mathbf{Z}}_{i}^{\top} \tilde{\mathbf{W}} \tilde{\mathbf{Z}}_{j}\right] \\
& =\mathrm{E}_{\mathbf{Z}_{j}}\left[\tilde{\mathbf{Z}}_{j}^{\top} \tilde{\mathbf{W}}^{\top} \mathrm{E}_{\mathbf{Z}_{i}}\left[\tilde{\mathbf{Z}}_{i} \tilde{\mathbf{Z}}_{i}^{\top}\right] \tilde{\mathbf{W}} \tilde{\mathbf{Z}}_{j}\right] \\
& =\mathrm{E}_{\mathbf{Z}_{j}}\left[\tilde{\mathbf{Z}}_{j}^{\top} \tilde{\mathbf{W}}^{\top}\left(\boldsymbol{\Sigma}_{i}+\tilde{\boldsymbol{\tau}}_{i} \tilde{\boldsymbol{\tau}}_{i}^{\top}\right) \tilde{\mathbf{W}} \tilde{\mathbf{Z}}_{j}\right] \\
& =\operatorname{Tr}\left(\tilde{\mathbf{W}}^{\top}\left(\boldsymbol{\Sigma}_{i}+\tilde{\boldsymbol{\tau}}_{i} \tilde{\boldsymbol{\tau}}_{i}^{\top}\right) \tilde{\mathbf{W}} \boldsymbol{\Sigma}_{j}\right)+\tilde{\boldsymbol{\tau}}_{j}^{\top} \tilde{\mathbf{W}}^{\top}\left(\boldsymbol{\Sigma}_{i}+\tilde{\boldsymbol{\tau}}_{i} \tilde{\boldsymbol{\tau}}_{i}^{\top}\right) \tilde{\mathbf{W}} \tilde{\boldsymbol{\tau}}_{j},
\end{aligned}
$$

where

$$
\boldsymbol{\Sigma}_{i}=\left(\begin{array}{cc}
\operatorname{var}\left(\mathbf{Z}_{i}\right) & \mathbf{0} \\
\mathbf{0} & 0
\end{array}\right), \forall i
$$

We have used the property that $\forall \mathbf{A}$ a matrix,

$$
\mathrm{E}\left[\tilde{\mathbf{Z}}_{j}^{\top} \mathbf{A} \tilde{\mathbf{Z}}_{j}\right]=\operatorname{Tr}\left(\mathbf{A} \operatorname{var}\left(\tilde{\mathbf{Z}}_{j}\right)\right)+\mathrm{E}\left[\tilde{\mathbf{Z}}_{j}\right]^{\top} \mathbf{A} \mathrm{E}\left[\tilde{\mathbf{Z}}_{j}\right] .
$$

In the following, and in order to simplify the notations, we denote:

$$
\tilde{\mathbf{E}}_{i}=\mathrm{E}_{\mathbf{Z}_{i}}\left[\tilde{\mathbf{Z}}_{i} \tilde{\mathbf{Z}}_{i}^{\top}\right]=\boldsymbol{\Sigma}_{i}+\tilde{\boldsymbol{\tau}}_{i} \tilde{\boldsymbol{\tau}}_{i}^{\top}
$$


Thus:

$$
\mathrm{E}_{\mathbf{Z}_{i}, \mathbf{Z}_{j}}\left[\left(\tilde{\mathbf{Z}}_{i}^{\top} \tilde{\mathbf{W}} \tilde{\mathbf{Z}}_{j}\right)^{2}\right]=\operatorname{Tr}\left(\tilde{\mathbf{W}}^{\top} \tilde{\mathbf{E}}_{i} \tilde{\mathbf{W}} \boldsymbol{\Sigma}_{j}\right)+\tilde{\boldsymbol{\tau}}_{j}{ }^{\top} \tilde{\mathbf{W}}^{\top} \tilde{\mathbf{E}}_{i} \tilde{\mathbf{W}} \tilde{\boldsymbol{\tau}}_{j}
$$

We eventually get the expression of a tractable second lower bound:

$$
\begin{aligned}
\mathcal{L}(q ; \boldsymbol{\alpha}, \tilde{\mathbf{W}}, \boldsymbol{\xi})=\sum_{i \neq j}^{N}\{ & \left(X_{i j}-\frac{1}{2}\right) \tilde{\boldsymbol{\tau}}_{i}^{\top} \tilde{\mathbf{W}} \tilde{\boldsymbol{\tau}}_{j}+\ln \mathrm{g}\left(\xi_{i j}\right)-\frac{\xi_{i j}}{2} \\
& \left.-\lambda\left(\xi_{i j}\right)\left(\operatorname{Tr}\left(\tilde{\mathbf{W}}^{\top} \tilde{\mathbf{E}}_{i} \tilde{\mathbf{W}} \boldsymbol{\Sigma}_{j}\right)+\tilde{\boldsymbol{\tau}}_{j}^{\top} \tilde{\mathbf{W}}^{\top} \tilde{\mathbf{E}}_{i} \tilde{\mathbf{W}} \tilde{\boldsymbol{\tau}}_{j}-\xi_{i j}^{2}\right)\right\} \\
& +\sum_{i=1}^{N} \sum_{q=1}^{Q}\left\{\tau_{i q} \ln \alpha_{q}+\left(1-\tau_{i q}\right) \ln \left(1-\alpha_{q}\right)\right\} \\
& -\sum_{i=1}^{N} \sum_{q=1}^{Q}\left\{\tau_{i q} \ln \tau_{i q}+\left(1-\tau_{i q}\right) \ln \left(1-\tau_{i q}\right)\right\} .
\end{aligned}
$$

with

$$
\ln p(\mathbf{X} \mid \boldsymbol{\alpha}, \tilde{\mathbf{W}}) \geq \mathcal{L}(q ; \boldsymbol{\alpha}, \tilde{\mathbf{W}}) \geq \mathcal{L}(q ; \boldsymbol{\alpha}, \tilde{\mathbf{W}}, \boldsymbol{\xi})
$$

APPENDIX B: OPTIMIZATION

\section{B.1. Optimization of $\xi_{i j}$.}

$$
\begin{aligned}
\frac{\partial \mathcal{L}}{\partial \xi_{i j}}(q ; \boldsymbol{\alpha}, \tilde{\mathbf{W}}, \boldsymbol{\xi})= & \mathrm{g}\left(-\xi_{i j}\right)-\frac{1}{2}-\lambda^{\prime}\left(\xi_{i j}\right)\left(\operatorname{Tr}\left(\tilde{\mathbf{W}}^{\top} \tilde{\mathbf{E}}_{i} \tilde{\mathbf{W}} \boldsymbol{\Sigma}_{j}\right)+\tilde{\boldsymbol{\tau}}_{j}{ }^{\top} \tilde{\mathbf{W}}^{\top} \tilde{\mathbf{E}}_{i} \tilde{\mathbf{W}} \tilde{\boldsymbol{\tau}}_{j}-\xi_{i j}^{2}\right) \\
& +2 \xi_{i j} \lambda\left(\xi_{i j}\right) \\
= & -\lambda^{\prime}\left(\xi_{i j}\right)\left(\operatorname{Tr}\left(\tilde{\mathbf{W}}^{\top} \tilde{\mathbf{E}}_{i} \tilde{\mathbf{W}} \boldsymbol{\Sigma}_{j}\right)+\tilde{\boldsymbol{\tau}}_{j}{ }^{\top} \tilde{\mathbf{W}}^{\top} \tilde{\mathbf{E}}_{i} \tilde{\mathbf{W}} \tilde{\boldsymbol{\tau}}_{j}-\xi_{i j}^{2}\right),
\end{aligned}
$$

where we have used the property that $(\operatorname{lng})^{\prime}\left(\xi_{i j}\right)=\mathrm{g}\left(-\xi_{i j}\right)$ and $\mathrm{g}\left(\xi_{i j}\right)+$ $\mathrm{g}\left(-\xi_{i j}\right)=1$. Since each bound $\ln \mathrm{g}\left(-a_{\mathbf{Z}_{i}, \mathbf{Z}_{j}}, \xi_{i j}\right)$ is an even function with respect to $\xi_{i j}$, we can consider only positive values of $\xi_{i j}$ without loss of generality. Therefore, we have $\lambda^{\prime}\left(\xi_{i j}\right) \neq 0$ since $\lambda\left(\xi_{i j}\right)$ is a strictly decreasing function on this domain. Finally, if we set the derivative (4) of the lower bound to zero, we obtain:

$$
{\hat{\xi_{i j}}}^{2}=\operatorname{Tr}\left(\tilde{\mathbf{W}}^{\top} \tilde{\mathbf{E}}_{i} \tilde{\mathbf{W}} \boldsymbol{\Sigma}_{j}\right)+\tilde{\boldsymbol{\tau}}_{j}^{\top} \tilde{\mathbf{W}}^{\top} \tilde{\mathbf{E}}_{i} \tilde{\mathbf{W}} \tilde{\boldsymbol{\tau}}_{j} .
$$




\section{B.2. Optimization of the class probabilities.}

$$
\frac{\partial \mathcal{L}}{\partial \alpha_{q}}(q ; \boldsymbol{\alpha}, \tilde{\mathbf{W}}, \boldsymbol{\xi})=\sum_{i=1}^{N}\left\{\frac{\tau_{i q}}{\alpha_{q}}-\left(\frac{1-\tau_{i q}}{1-\alpha_{q}}\right)\right\}=0
$$

Thus,

$$
\left(1-\alpha_{q}\right) \sum_{i=1}^{N} \tau_{i q}=\alpha_{q} \sum_{i=1}^{N}\left(1-\tau_{i q}\right)
$$

This leads to

$$
\sum_{i=1}^{N} \tau_{i q}=\alpha_{q} N
$$

and

$$
\hat{\alpha_{q}}=\frac{\sum_{i=1}^{N} \tau_{i q}}{N} .
$$

\section{B.3. Optimization of $\tilde{\mathbf{W}}$.}

$\nabla_{\tilde{\mathbf{W}}} \mathcal{L}(q ; \boldsymbol{\alpha}, \tilde{\mathbf{W}}, \boldsymbol{\xi})=\sum_{i \neq j}^{N}\left\{\left(X_{i j}-\frac{1}{2}\right) \tilde{\boldsymbol{\tau}}_{i} \tilde{\boldsymbol{\tau}}_{j}{ }^{\top}-2 \lambda\left(\xi_{i j}\right)\left(\tilde{\mathbf{E}_{i}} \tilde{\mathbf{W}} \boldsymbol{\Sigma}_{j}+\tilde{\mathbf{E}}_{i} \tilde{\mathbf{W}} \tilde{\boldsymbol{\tau}}_{j} \tilde{\boldsymbol{\tau}}_{j}{ }^{\top}\right)\right\}$,

since $\forall \mathbf{B}, \mathbf{C}$ symmetric matrices:

$$
\nabla_{\tilde{\mathbf{W}}} \operatorname{Tr}\left(\tilde{\mathbf{W}}^{\top} \mathbf{B} \tilde{\mathbf{W}} \mathbf{C}\right)=\mathbf{B} \tilde{\mathbf{W}} \mathbf{C}+\mathbf{B}^{\top} \tilde{\mathbf{W}} \mathbf{C}^{\top}=2 \mathbf{B} \tilde{\mathbf{W}} \mathbf{C},
$$

and $\forall \mathbf{b}$ a vector:

$$
\nabla_{\tilde{\mathbf{W}}} \mathbf{b}^{\top} \tilde{\mathbf{W}}^{\top} \mathbf{B} \tilde{\mathbf{W}} \mathbf{b}=\mathbf{B}^{\top} \tilde{\mathbf{W}} \mathbf{b} \mathbf{b}^{\top}+\mathbf{B} \tilde{\mathbf{W}} \mathbf{b} \mathbf{b}^{\top}=2 \mathbf{B} \tilde{\mathbf{W}} \mathbf{b} \mathbf{b}^{\top} .
$$

Finally, we obtain:

$$
\nabla_{\tilde{\mathbf{W}}} \mathcal{L}(q ; \boldsymbol{\alpha}, \tilde{\mathbf{W}}, \boldsymbol{\xi})=\sum_{i \neq j}^{N}\left\{\left(X_{i j}-\frac{1}{2}\right) \tilde{\boldsymbol{\tau}}_{i} \tilde{\boldsymbol{\tau}}_{j}{ }^{\top}-2 \lambda\left(\xi_{i j}\right) \tilde{\mathbf{E}}_{i} \tilde{\mathbf{W}} \tilde{\mathbf{E}}_{j}\right\} .
$$

Therefore, the matrix $\tilde{\mathbf{W}}$ which maximizes the lower bound satisfies:

$$
2 \sum_{i \neq j}^{N} \lambda\left(\xi_{i j}\right) \tilde{\mathbf{E}}_{i} \tilde{\mathbf{W}} \tilde{\mathbf{E}}_{j}=\sum_{i \neq j}^{N}\left(X_{i j}-\frac{1}{2}\right) \tilde{\boldsymbol{\tau}}_{i} \tilde{\boldsymbol{\tau}}_{j}{ }^{\top}
$$

This implies:

$$
\operatorname{vec}\left\{2 \sum_{i \neq j}^{N} \lambda\left(\xi_{i j}\right) \tilde{\mathbf{E}}_{i} \tilde{\mathbf{W}} \tilde{\mathbf{E}}_{j}\right\}=\operatorname{vec}\left\{\sum_{i \neq j}^{N}\left(X_{i j}-\frac{1}{2}\right) \tilde{\boldsymbol{\tau}}_{i} \tilde{\boldsymbol{\tau}}_{j}^{\top}\right\},
$$


and

$$
2 \sum_{i \neq j}^{N} \lambda\left(\xi_{i j}\right) \operatorname{vec}\left(\tilde{\mathbf{E}_{i}} \tilde{\mathbf{W}} \tilde{\mathbf{E}}_{j}\right)=\sum_{i \neq j}^{N}\left(X_{i j}-\frac{1}{2}\right) \operatorname{vec}\left(\tilde{\boldsymbol{\tau}}_{i} \tilde{\boldsymbol{\tau}}_{j}^{\top}\right),
$$

where vec denotes an operator which stacks the columns of a matrix into a vector. From (7), we obtain:

$$
2 \sum_{i \neq j}^{N} \lambda\left(\xi_{i j}\right)\left(\tilde{\mathbf{E}}_{j} \otimes \tilde{\mathbf{E}}_{i}\right) \operatorname{vec}(\tilde{\mathbf{W}})=\sum_{i \neq j}^{N}\left(X_{i j}-\frac{1}{2}\right)\left(\tilde{\boldsymbol{\tau}}_{j} \otimes \tilde{\boldsymbol{\tau}}_{i}\right),
$$

since $\tilde{\mathbf{E}}_{j}$ is a symmetric matrix and $\forall \mathbf{B}, \mathbf{C}$ two matrices:

$$
\operatorname{vec}(\mathbf{B} \tilde{\mathbf{W}} \mathbf{C})=\left(\mathbf{C}^{\boldsymbol{\top}} \otimes \mathbf{B}\right) \operatorname{vec}(\tilde{\mathbf{W}})
$$

Moreover $\forall \mathbf{b}$, $\mathbf{c}$ two vectors:

$$
\operatorname{vec}\left(\mathbf{c} \mathbf{b}^{\top}\right)=\mathbf{b} \otimes \mathbf{c} .
$$

Therefore an estimate of $\operatorname{vec}(\tilde{\mathbf{W}})$ is given by:

$$
\begin{gathered}
\operatorname{vec}(\tilde{\mathbf{W}})=\left\{2 \sum_{i \neq j}^{N} \lambda\left(\xi_{i j}\right)\left(\tilde{\mathbf{E}}_{j} \otimes \tilde{\mathbf{E}}_{i}\right)\right\}^{-1}\left\{\sum_{i \neq j}^{N}\left(X_{i j}-\frac{1}{2}\right)\left(\tilde{\boldsymbol{\tau}}_{j} \otimes \tilde{\boldsymbol{\tau}}_{i}\right)\right\} . \\
\text { REFERENCES }
\end{gathered}
$$

\section{REFERENCES}

JAAKKOLA, T. S. and Jordan, M. I. (2000). Bayesian parameter estimation via variational methods. Statistics and Computing 10 25-37.

Laboratoire Statistique et GÉnome UMR CNRS 8071, INRA 1152, UEVE 91000 Evry, France

E-MAIL: pierre.latouche@genopole.cnrs.fr etienne.birmele@genopole.cnrs.fr
Laboratoire Statistique et Génome UMR CNRS 8071, INRA 1152, UEVE 91000 Evry, France

E-MAIL: christophe.ambroise@genopole.cnrs.fr 